Review

\title{
Educational Challenges in Computer-based Finite Element Analysis and Design of Structures
}

\author{
Vagelis Plevris and Gro Markeset \\ Department of Civil Engineering and Energy Technology, OsloMet-Oslo Metropolitan University, Oslo, Norway
}

Article history

Received: 01-07-2018

Revised: 06-09-2018

Accepted: 24-10-2018

Corresponding Author:

Vagelis Plevris

Department of Civil

Engineering and Energy

Technology, OsloMet-Oslo

Metropolitan University, Oslo,

Norway

Email: vageli@oslomet.no

\begin{abstract}
Computer simulations and computational methods, such as the Finite Element Analysis (FEA) have become essential methodologies in science and engineering during the last decades, in a wide variety of academic fields. Six decades after the invention of the digital computer, advanced FE simulations are used to enhance and leapfrog theoretical and experimental progress, at different levels of complexity. Particularly in Civil and Structural Engineering, significant research work has been made lately on the development of FE simulation codes, methodologies and validation techniques for understanding the behavior of large and complex structures such as buildings, bridges, dams, offshore structures and others. These efforts are aimed at designing structures that are resilient to natural excitations (wind loads, earthquakes, floods) as well as human-made threats (impact, fire, explosion and others). The skill set required to master advanced FEA is inherently interdisciplinary, requiring in-depth knowledge of advanced mathematics, numerical methods and their computational implementation, as well as engineering sciences. In this paper, we focus on the importance of sound and profound engineering education and knowledge about the theory behind the Finite Element Method to obtain correct and reliable analysis results for designing real-world structures. We highlight common mistakes made by structural engineers while simulating complex structures and the risk of structural damage because of humanmade mistakes or errors in the model assumptions. The event of the collapse and eventual sinking of a concrete offshore platform in the North Sea is presented as a case study where a serious error in the finite element analysis played a crucial role leading to structural failure and collapse.
\end{abstract}

Keywords: Computer Methods, Engineering Education, Finite Element Method, Finite Element Analysis, FEM, FEA, Simulation Error, Modeling Error, Structural Damage

\section{Introduction}

Finite Element Analysis (FEA) is a computer method used to analyze engineering structures. FEA is used in many different engineering disciplines such as solid mechanics, thermodynamics (heat transfer), fluid dynamics (fluid flow), electrostatics and others. In the present work, we focus on structural and continuum mechanics, which are the main application areas of FEA in Civil and Structural Engineering. In this discipline, the method can be used to calculate the displacements, stresses, dynamic behavior, buckling behavior of structures, among many others (Plevris and Tsiatas, 2018).

The method is based on the division of the structure into small parts (elements) where a set of simple equations can describe the behavior of each element. The Finite Element Method (FEM) uses the concept of piecewise polynomial interpolation: By connecting elements together, the field quantity becomes interpolated over the entire structure in a piecewise fashion. Each element contributes stiffness to the whole structure and by combining the stiffness from all elements, the model stiffness matrix $[K]$ is formed also taking into account the geometry, the constraints and the connectivity between the elements. In linear analysis, by specifying the load vector $\{F\}$, the method results in a set of simultaneous algebraic equations, which are solved for the displacements $\{u\}$ at the nodal points, as shown in Equation: 


$$
[K]\{u\}=\{F\} \Rightarrow\{u\}=[K]^{-1}\{F\}
$$

Once having calculated the nodal displacements, it is easy to calculate other derived quantities such as stresses, bending moments, shear and axial forces, intermediate displacements and others according to the type of the structure and the types of elements used. The results of FEA can be easily depicted graphically on the computer screen with nice visualizations using color contour plots, animations and others.

Although in the present paper we focus mainly on structural engineering and elasticity problems, FEA can also be applied to other disciplines and the main philosophy of the method remains unchanged. Table 1 shows various applications of FEA in different disciplines and the meanings or $[\mathrm{K}],\{\mathrm{u}\}$ and $\{\mathrm{F}\}$ for every case.

FEA is very useful in cases where performing hand calculations would not provide accurate results due to the complexity of the analysis problem. FEA can also replace expensive experiments as numerical simulations have a trivial cost in comparison to real-life tests where a structure must be put under increasing stress, get damaged or even destroyed. FEA can provide an accurate and inexpensive alternative to such tests.

\section{Literature Review}

Although FEA is an issue of tremendous significance in engineering practice today, its teaching is quite demanding and difficult. The method by its nature requires a very good knowledge of elasticity theory, matrix algebra, mechanics and also computer programming skills to various degrees. It is a challenge for a FEA educator to teach it to an undergraduate or graduate audience in such a way that the learning outcomes will be achieved and students will enjoy and appreciate the course. (Hossain, 2015) presented the pedagogical approaches undertaken to successfully implement a FEA course objectives to undergraduate engineering students, addressing the topics and techniques applied to teach different concepts of FEA to enhance students learning outcomes. The work of (Zamani, 2016) highlights the challenges in teaching FEA to undergraduate students due to the widespread availability of commercial FEA software in the academic institutions and the pressure from industry to include it in the modern undergraduate curriculum. The role of commercial software in FEA teaching at the undergraduate level is also investigated by (Shaikh, 2012). In this work it was observed that the use of commercial software can greatly help students' understanding of FEA. Young et al. (2012) presented the results of a four-year study using 3D FEA visualization of structures for teaching in civil engineering, concluding that $3 \mathrm{D}$ visualization of structures using FEA can be used as an effective teaching tool for civil engineering students in structural analysis courses.

\begin{tabular}{llll}
\multicolumn{4}{l}{ Table 1: FEA disciplines and the meanings of $[K],\{u\}$ and $\{F\}$} \\
\hline Discipline & Property $[\mathrm{K}]$ & Behavior $\{\mathrm{u}\}$ & Action $\{\mathrm{F}\}$ \\
\hline Elastic & Stiffness & Displacement & Force \\
Thermal & Conductivity & Temperature & Heat source \\
Fluid & Viscosity & Velocity & Body force \\
Electrostatic & Dielectric & Electric & Charge \\
& permittivity & potential & \\
\hline
\end{tabular}

In the present work, we highlight possible sources of error in FEA, common mistakes made by structural engineers while simulating complex structures, errors in the model assumptions and the risk of structural damage because of careless FEA use. The paper attempts to make a useful contribution to the vital discussion on FEA engineering education at present and therefore focuses on the importance of sound and profound engineering education and knowledge about the theory behind the Finite Element Method to obtain correct and reliable analysis results for analyzing and designing realworld structures. We emphasize that the key to good FE analysis is the knowledge of the limitations of the method and an understanding of the physical phenomena under investigation. The event of the collapse and eventual sinking of a concrete offshore platform in the North Sea is presented as a case study where a serious error in the finite element analysis played a crucial role leading to structural collapse.

\section{Advantages and Disadvantages of FEA}

As any similar numerical method, FEA exhibits advantages and disadvantages and can even produce errors when not used correctly. The main advantages and disadvantages of the method are summarized below.

\section{Advantages}

- Has a wide range of applications: aerospace, civil, mechanical, electronics and many more

- Can readily handle very complex geometries

- Can handle a wide variety of problems

- Can handle complicated restraints (supports), loadings and/or materials

- $\quad$ Saves time, money and effort to conduct empirical studies to analyze new scenarios

- $\quad$ FEA programs offer great pre- and post-processing facilities nowadays

- Rapid calculation time for most applications. As computer power keeps increasing, FEA becomes even more available, faster and easier to use

\section{Disadvantages}

- A general closed-form mathematical solution cannot be obtained

- The solution is only an approximation - the analysis is not performed on the real structure, but on a model of it. As a result, the analysis has inherent errors 
- The output is only as good as the input. User mistakes can be dangerous or even fatal

- $\quad$ FEA programs can be very expensive, complex and hard to master, especially in specialized nonlinear analysis

\section{The Risk of Using FEA Carelessly - GIGO Principle}

Numerous public-domain, free or commercial packages are presently available for FEA. Commonly, FEA software comes together with fancy $3 \mathrm{D}$ pre- and post-processors for visualizing the model and the analysis results. Never in the past had the modeling and analysis of complex structures been more accessible than today. Many FEA software houses claim that modeling using their programs is very easy, implying that even an inexperienced user (or a non-engineer!) can easily become a FEA expert producing simulation models and FEA results (Kurowski, 2002). Others consider FEA as just a simple extension of Computer Aided Design (CAD) thinking that good FE modeling is only a matter of the accurate representation of the geometry of the structure when in fact the similarity between CAD and FEA is only there; in the geometry. Other than geometry, in FEA, meshing, element type, loads, supports, error estimation and analysis results interpretation and validation are critical issues that have very little to do with CAD.

FEA can reduce the need for hand calculations significantly. However, one still needs to make a final confirmation of the model, validate the results and justify the answers. It is rather easy to generate a goodlooking 3D model with colorful visualizations of the model or the analysis results, but there is always the risk that results may be misleading or even downright wrong. Often people also think that a mesh refinement and a more powerful computer can solve all problems, but again this is an over-simplification and not the case in reality. One can be easily misled into blind acceptance of answers produced by the program. In the end, in FEA, the GIGO principle ("Garbage In $\rightarrow$ Garbage Out") fully applies. GIGO is a computer science acronym that implies bad input will always result to bad output, referring to the fact that computers will unquestioningly process flawed, even nonsensical input data and produce undesired, often meaningless output if mishandled.

It is imperative not to confuse pretty graphs and colorful model pictures with correct modeling practice and accurate results. In many cases, preliminary calculations can tell us that something is wrong or suspicious. Many experts suggest that modelers should also perform simple hand calculations to get an idea of the order of magnitude of the anticipated results. When the analysis results are orders of magnitude different from the hand calculation results of a simplified model, then one has an alarm that something is wrong and needs to be corrected.

A successful FEA project requires proper execution of the following three complex processes: (i) The analyst must be capable and qualified to pose a "question" correctly to the software

(ii) The software must be mathematically robust and accurate enough to provide a reasonable and accurate solution

(iii) The analyst must be qualified to understand the results and assess the performance of the structural system based on these results

To achieve the above, the engineer/designer needs to have the following skills:

(i) Excellent knowledge of the FEA theory and strong engineering judgment

(ii) Facility with the FEA software being used

(iii) Good practical modeling experience and a feel for design

While the second and third can be obtained to a certain extent through professional experience in structural analysis and design consulting firms that use FEA, the first is particularly critical in making the right assumptions in the model. These assumptions include which features should be modeled; which are not so important to model; what model parameters are important; how to correctly apply loads and constraints; how to correctly interpret the results, among others. Understanding the FE method is more important than specific software commands, which can be quickly learned even by non-experts. It is a much deeper issue, which requires excellent university education in FEA, usually at a post-graduate level. Inadequate FEA education and training poses risks and produces hazards.

In some cases, the only training one has comes from software vendors through informative seminars and presentations. Such instruction may be superficial and concentrate only on how to run a specific software rather than on really understanding FEA. Such seminars are useful and can offer complementary knowledge on the use of a specific program, but they can never be enough on their own.

\section{Possible Sources of Error in FEA}

FEM is comprised of three major phases: (i) Preprocessing, in which the analyst sets up the model, develops a FE mesh to divide the subject geometry into subdomains for mathematical analysis and applies material properties, boundary conditions and analysis options; (ii) Analysis, during which the program derives the governing matrix equations from the model, solves for the nodal displacements and calculates derived quantities; and (iii) Post-processing, in which the analyst checks the validity of the solution, examines the values of primary quantities (such as displacements and stresses) and derives and examines additional quantities (such as specialized stresses and error indicators). 
Table 2: Possible sources of error in Finite Element Analysis

A. Human (Engineer)

1. Pre-processing A1-1. Wrong/bad engineering assumptions

A1-2. Wrong/bad type of elements

A1-3. Discretization error/Inappropriate or bad mesh

A1-4. Wrong releases in beam elements

A1-5. Connectivity problems

2. Analysis

3. Post-processing

B. FEA Software

1. Pre-processing

2. Analysis

3. Post-processing
A2-1. Human mistakes

A2-2. Wrong boundary conditions (supports)

A2-3. Wrong analysis method or analysis options

A2-4. Part of the model is a mechanism

A3-1. Wrong interpretation of the coordinate system

A3-2. Inability to distinguish between important and non-important findings A3-3. Units' mix-up or misinterpretation

B1-1. Bad element formulation

B1-2. Bad meshing algorithm

B2-1. Inaccurate analysis

B2-2. Numerical Errors

B3-1. Wrong averaging of results

B3-2. Bad visualization of results

B3-3. No user warnings on obvious errors
The linear FEA study simulates reality only when a number of restricting conditions are met. These include that all deflections are infinitesimally small and that stresses and strains induced by loads remain small enough to let their linear relationship hold (Hooke's law). In reality, almost all problems are nonlinear, either from the beginning or at higher load levels. Nonlinearities can vary, including nonlinear material laws, nonlinear geometry, problems with instabilities, contact problems and others. In nonlinear FEA (NL-FEA) the principle of superposition cannot be applied; the loading history may be significant and the analysis phase gets much more complicated as it is conducted iteratively and not in a single load step. In the present study, we focus primarily on linear FEA, although many of the remarks and findings of the work have a general nature and certainly apply also to nonlinear FEA procedures.

The errors in a FE analysis can be divided into two parts: Error made by the engineer and error made by the FEA software (Nelson and Wang, 2004). Nowadays FEA programs have gradually become more advanced and the problems of the first generations of programs have been more or less fixed. Thus, the most crucial cause of error presently is the human-made error. Table 2 summarizes the most common sources of error.

\section{Human-Made Errors (Engineer)}

This section explains the various human-made errors that are described in Table 2.

\section{Pre-Processing Phase}

\section{A1-1. Wrong/Bad Engineering Assumptions}

The assumptions made by the engineer are significant for the accurate modeling of the structure and the validity of the analysis results. Incorrect modeling assumptions can lead to a situation where predictions made using the model may differ significantly from the actual structural behavior (Bandara et al., 2009). The assumptions have to do with various properties of the model: Assumptions about loads and boundary conditions, material properties, geometric characteristics, material behavior, needed analysis type (linear, nonlinear, etc.) and many others.

\section{A1-2. Wrong/Bad Type of Elements}

Such modeling errors have to do with simplifying assumptions in the mathematical model. Most commonly, they have to do with the assumptions of the element being used. Below are a few typical examples of such cases:

(i) In beam elements, a major assumption is that crosssections stay planar and do not change shape, which is not always the case in practice

(ii) Shell elements also have limitations (having to do with the "drilling" degrees of freedom) and, in some cases, they need to be replaced by solid elements to be able to catch the real structural behavior

(iii) Shear stresses are not calculated for the commonly used Bernoulli-type beam elements, but only for Timoshenko-type beam elements. The engineer has to understand the problem at hand and use the right type of elements to deal with it, while choosing more complex elements is not always the best strategy, depending again on the problem

(iv) Use of plane stress/plane strain elements where zero out of plane stress/strain is assumed, respectively; the inherent assumptions must be checked carefully

Another important phenomenon that has to do with the selection of the element type as well as the mesh, 
requiring special attention and care, is shear locking. Shear locking is an artifact in structural calculations by FEM which arises due to the inability of an element to capture the kinematics of deformation. Locking can occur for several reasons and, for some element types, can even depend on the shape of an element. In general, locking happens when an element cannot interpolate a field property correctly with the nodal values and the element's shape functions (Macneal, 1993). In FEA terms, shear locking causes the element to behave stiffer than what it actually is. This behavior is observed only in linear elements (for example the constant strain triangle - CST), i.e., elements that use a linear interpolation function for the displacement, as strain captured in a linear element is a constant strain. In cases with such problems, a mesh refinement can help or even better the use of higher order elements that do not suffer from shear locking.

\section{A1-3. Discretization Error/Inappropriate or Bad Mesh}

Although there are quite a few mesh-free (or meshless) FEA codes, meshing is still one of the most essential tasks for many FEA users. Discretization errors have to do with poor discretization of the domain, i.e., inappropriate or bad mesh. In some areas of the domain (e.g., in regions with high stress or strain gradients), a finer mesh may be needed to end up with good analysis results. The engineer needs to identify these areas and take proper measures to make sure that the mesh is adequate for the representation of the local displacements and stresses. On the other hand, a general mesh refinement (i.e., more dense mesh) is not always a better modeling strategy and can even lead to other types of problems. Also, bad-shaped or distorted elements must be avoided. Examples of elements with good and bad aspect ratios are presented in Fig. 1.

\section{A1-4. Wrong Releases in Beam Elements}

In civil engineering, it is common to use onedimensional beam elements to model columns, beams or other linear elements of a frame structure. In usual cases, the connection between elements is solid (or "fixed") which means that all displacements and rotations are common between the two connecting elements at the location of the joint (common node). In some cases, mostly in steel structures, special circumstances apply and releases need to be used at the elements' ends, most commonly hinges (rotational releases). It is the responsibility of the engineer to use the right releases in every case as wrong releases can have a dramatic effect on the analysis results.
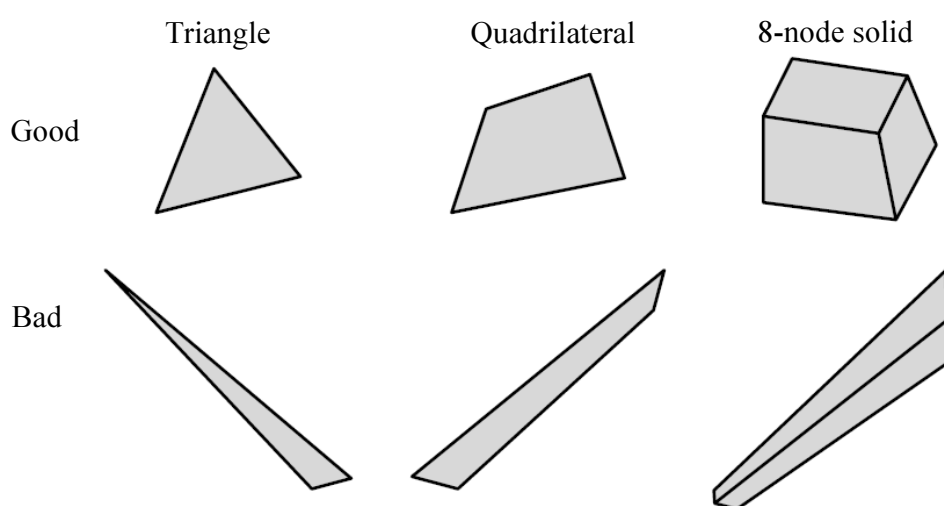

Fig. 1. Elements with good and bad aspect ratios

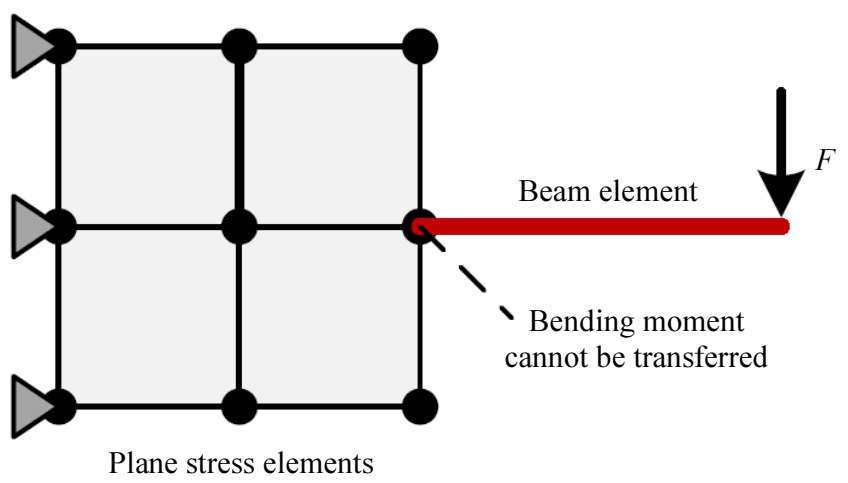

Fig. 2: Bad connection: Incorrect coupling of elements with different DOFs 


\section{A1-5. Connectivity Problems}

This problem has to do with elements that appear to be connected visually, but in practice, there is no real connection. One has to be very careful, as this kind of problem is problematic to identify on the computer screen. Moreover, special care has to be taken when different types of elements are connected. For example, a common problem is the case of a beam element connected to plane stress elements where the moment cannot be transferred between the two types of elements, as shown in Fig. 2.

The same problem arises when frame elements (beams) are connected to solid objects that have only three translational DOFs, therefore they provide no rotational resistance to the interconnected frame objects. To handle such problems, one needs to apply tricks and smart modeling techniques, for example: (i) extend ("anchor") the beam inside the plane/solid elements; (ii) apply a body constraint or rigid link that will connect the end joint of the beam to the tributary joints of the plane/solid elements such that a force couple is available to resist moment within the frame joint; (iii) in the plane elements case, use other types of elements that are also capable of transmitting the moment from the beam (drilling DOF). However, the stiffness properties of the drilling DOFs, which are normal to shell surfaces, are in general not reliable for the connection of frame objects. As the local mesh is refined, the connection can become increasingly flexible, so this solution must be used with care and attention.

\section{Analysis Phase}

\section{A2-1. Human Mistakes}

Mistakes such as forgetting an important load, mistakes in the units used, the thickness of beams or the orientation of the elements, loads wrong in location, type, direction or magnitude, etc. can cause fatal problems in the FE analysis. The engineer must be meticulous in every step of the modeling and analysis phase and has to double check the model data to ensure that everything is modeled and entered correctly in the program.

\section{A2-2. Wrong Boundary Conditions (Supports)}

Supports are commonly modeled with constrained DOFs that are either fully restrained (fixed DOFs) or have an elastic constant applied to them (spring DOFs), with given stiffness. Modeling problems have to do with supports being wrong in location, type or direction. If the number of supports on a model are insufficient, then a rigid body motion can be allowed leading to a mechanism. Supports should be sufficient to prevent all possible rigid-body motions. Such a problem is depicted in Fig. 3. While the modeling error is evident in this case, there are still cases where such rigid body motions in parts of the model are not so easy to spot.

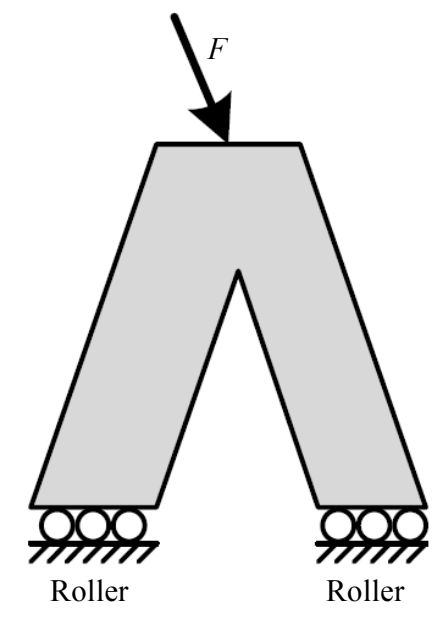

Fig. 3: Rigid body motion due to insufficient supports

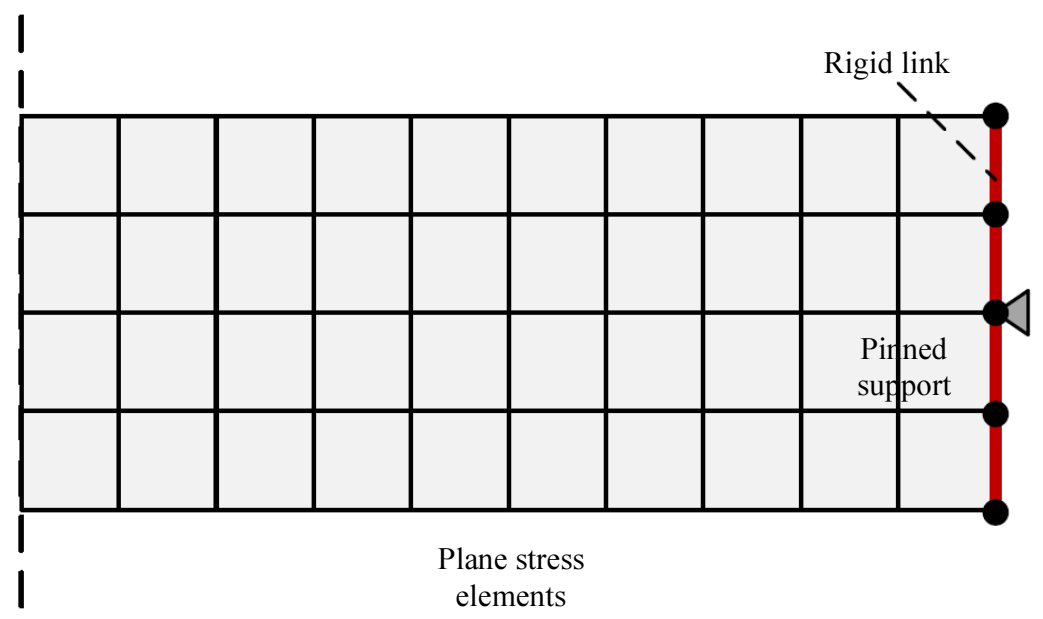

Fig. 4: Beam modeling with plane stress elements: Pinned support with use of a rigid link 
Applying specific supports may become tricky when using plane stress or solid elements to model beams. Such elements have only translational DOFs so it is not easy to deal with the beam rotation. In this case special modeling techniques have to be used, for example rigid links as shown in Fig. 4 where a beam, pinned at one end, is modeled using plane stress elements. Similar techniques can be used also for the case of beam modeling with $3 \mathrm{D}$ solids.

\section{A2-3. Wrong Analysis Method or Analysis Options}

This is important especially in nonlinear analysis where additional parameters need to be input, such as the number of iterations for convergence, convergence criteria, etc. Using the wrong calculation discipline can also be a problem, for example, when a problem needs nonlinear analysis (material and/or geometric nonlinearity) and a simple linear analysis is only performed. Furthermore, in some cases, the engineer has to select between static or various types of dynamic analyses correctly. If dynamic analysis is to be performed, various additional parameters have to be carefully set, such as the number of eigen modes taken into account, or the values of the various constants of the time integration algorithm to be used.

\section{A2-4. Part of the Model is a Mechanism}

Because of various types of errors, the structure or parts of it can become a mechanism. Practically the stiffness matrix of the structure cannot be inverted (matrix determinant equal to zero). In some cases, the program will experience a runtime error and will inform the user about it. However, there are cases where due to numerical approximation errors, the program manages to provide a false "solution" without displaying any error message, due to rounding errors. In that case, displacements will have extreme values while the results may not correspond to an equilibrium of the model. The user should be able to identify such problematic results even if the computer program does not provide any warning message.

\section{Post-Processing Phase}

\section{A3-1. Wrong Interpretation of the Coordinate System}

The program provides the output values, but the user also needs to be able to understand these values and their true meaning. It is common that the user is not aware of the coordinate system that the program uses to report its analysis results, which may lead to misinterpretation of the results. A careful reading of the documentation of the program is suggested to deal with such problems.

\section{A3-2. Inability to Distinguish Between Important and Non-Important Findings}

The amount of information that can be produced by a FEA software, especially for nonlinear analysis, is enormous. The engineer needs to be able to assess the importance of the FEA results. For example, high values of stresses locally near a point load should not be taken into account quantitatively. If one is not aware of this and only considers the maximum stress, then the results can be interpreted the wrong way. According to the wellknown Saint-Venant's Principle (Timoshenko and Goodier, 1951) loads applied in a finite area will produce local stresses and strains but as a far as the rest of the structure is concerned, the local effects will not be experienced and the load could be treated as a point load. In other words, the differences between the effects of two different but statically equivalent loads become very small at sufficiently large distances from the load. However, this rule does not apply to every type of member and loading case. For example, loaded members made from thin-walled elements may create stresses and deformations that can have an influence a considerable distance away from the point of application of loading, due to nonlinear buckling (Hibbeler, 1994).

\section{A3-3. Units' Mix-Up or Misinterpretation}

It is crucial to understand units, how they work and how the program treats them. Some programs are unitless (solving the purely mathematical problem without applying any unit conversions to inputs or outputs), only requiring the user to use consistent units for inputs and then the program outputs will comply. Others provide units and apply automatic unit conversion factors on inputs and outputs. If units are not defined the right way in the model data, then the results will be all wrong. Similarly, correct results may be interpreted the wrong way if the designer cannot realize what units the program actually reports in.

\section{FEA Software Made Errors}

FEA software made errors are no longer prevalent as FEA software packages have improved significantly over the time and keep improving continuously. Program bugs have existed and will always exist, but most commercial FEA packages nowadays have been tested and validated thoroughly by thousands of users on several problems and various situations. Again, it is the user's responsibility to use FEA software that is tested, validated and considered reliable for one's analysis needs.

While the Pre- and Post-processing phases of FEM are interactive and time-consuming for the analyst, the analysis phase (case B2-1 of Table 2) is often a batch process which is quite demanding in computer resources. Presently the main commercial FE programs are verified to a certain extent and their analysis results can be considered accurate. Attention must be paid when using new software from unknown vendors. It is always better to use software that has been used by many users in various situations and can be considered trustworthy. 


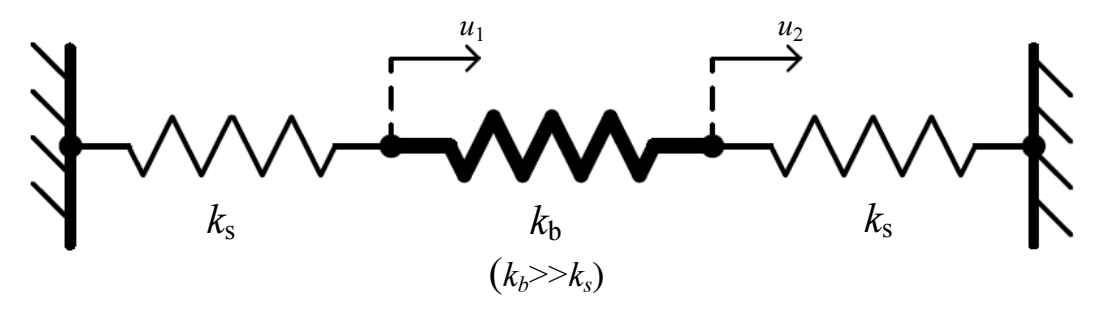

Fig. 5: A simple system of three springs in series

Numerical errors (case B2-2) can occur due to the limited number of significant digits essentially maintained by the computer. These rounding errors can even accumulate, especially in large models leading to errors in the results, although this is not so common to happen. Similar numerical problems can occur if there are large differences in the elements' stiffness. In this case, the results cannot be trustworthy. Even in the simple example problem of three springs connected in series, shown in Fig. 5, if there is a significant difference in the stiffness of the springs (e.g., $k_{b}>>k_{s}$ ) then numerical problems can occur (ill-conditioned system).

The stiffness matrix of the above system is:

$K=\left[\begin{array}{cc}k_{b}+k_{s} & -k_{b} \\ -k_{b} & k_{b}+k_{s}\end{array}\right]$

Moreover, since $k_{b}>>k_{s}$ then $k_{b}+k_{s} \approx k_{b}$ and as a result:

$K \approx\left[\begin{array}{cc}k_{b} & -k_{b} \\ -k_{b} & k_{b}\end{array}\right]$

The stiffness matrix shown in Equation (3) is apparently not reversible (its determinant equals zero) and this proves that significant differences in the stiffness of elements can cause numerical problems in the analysis even in simple problems like this. In fact, this is not a limitation of the software or the method, but rather an inherent mathematical problem that the user has to be aware of. Such substantial difference in stiffness should not be present in a real model.

User warnings on errors (case B3-3) are particularly important in a FEA software. The software must guide the user and clearly point out obvious problems and errors to him/her. Self-validation of the analysis and user warnings for all kinds of possible errors are necessary to decrease the risk of wrong analysis results.

\section{FEA Results Verification - Check List}

After the analysis, the FE results need always to be checked and validated. It is important to remember that even if the model is sound and everything appears to be perfect, FEA solvers always give approximate solutions. The following checklist includes various checks that need to be done in the pre- and post-processing phase. The list is indicative and not exhaustive.

\section{Assumptions}

Are the results consistent with the major model assumptions, e.g., small deformation or small strain?

\section{Dimensions}

Do key model dimensions agree with the drawings?

\section{Materials}

Are the material properties correct and are they adequately assigned to the various model regions?

\section{Element Properties}

Are element properties (shell thickness, beam properties) correct and adequately associated to the model regions?

\section{Mesh}

Do the elements have correct aspect ratios? Do they pass shape distortion testing criteria? Is the mesh sufficiently refined to produce the required accuracy in specific regions? Has the model been verified for meshsensitivity?

\section{Loads}

Are the applied loads correct in location, magnitude and direction? A standard error is the wrong coordinate system for loads, especially when importing loads from other sources.

\section{Displacements}

Are deflections symmetric in a symmetric problem? Are there large displacements that cause force directions to change or are there other 2 nd order phenomena? A nonlinear analysis would be needed in these cases.

\section{Supports}

Are the correct DOFs restrained? Do deflections obey intended support conditions? Do reaction forces agree with static calculations? Do they balance the applied loads in each direction for every load case? 


\section{Stresses}

Are stresses continuous across elements? Are stress contours normal to planes of symmetry? Are principal stresses zero along unloaded boundaries? Are the stress contours smooth in highly stressed areas?

\section{Hand Calculations}

Do deformations and stresses compare well with hand calculations for an equivalent yet much simpler model? Verify that the model represents the physical system by comparing the model to hand calculations, test data or other known sources. This verifies primarily the assumptions and abstractions made during modeling.

\section{Eigenfrequency Analysis}

Even if the problem is static (not dynamic), performing an eigenfrequency analysis and observing the eigenmodes (especially the 1st main mode) can help spot misconnections and other errors.

\section{Repetition}

Don't rely on a single simulation/analysis run. Refine the mesh in areas of high stress and repeat the analysis the needed number of times. Make experiments by changing key model properties to see how the results are affected and if the structural behavior is consistent with the change made, in every single case.

\section{FEA in Engineering Education and Teaching}

Initially, in most universities, finite element methods were only taught at the post-graduate level. As FEA codes became more generally available, user-friendly and easy to use, the teaching of FEM and FEA gradually found its way into undergraduate programs, also (Gilewski, 2008). The teaching of FEA is difficult by definition. The theory of the method involves difficult advanced mathematics, while to understand the method deeper, one also needs programming skills and an excellent command of computer usage. Usually, students prefer practical computer lessons with the use of userfriendly finite element commercial programs instead of lectures on FEM theory. The role of commercial software in teaching finite element analysis is essential and has been investigated by researchers (Shaikh, 2012). Nevertheless, in reality, it is not possible to use any FEM system efficiently without the base knowledge of the theory. The correct balance between the two can be tricky and is always a significant challenge for FEA university educators (Gilewski, 2008). Due to the wide acceptance and use of FEA for any built structure nowadays, it is of paramount importance that engineers have received a proper FEA university education. Lack of such knowledge can have dramatic consequences in the built environment and to the society as a whole.
In the authors' opinion, a finite element course should first try to introduce the students to the FE based modeling and analysis techniques currently being used in engineering practice. In the end, the students should be able to develop an appreciation for the underlying theory and its application. Teaching should involve a combination of theoretical lectures on FEM, computer lab tutorials and a compulsory project assignment. The reason for introducing fundamental finite element theory in a course is to avoid the danger of using FEA as only a "black box". The tutorials and assignment exercises reinforce an understanding of mechanics of materials theory, finite element theory, knowledge of physical behavior and usage of each element type, the ability to select a suitable element for a given problem and the ability to interpret and evaluate the quality of finite element results.

On the other hand, the project assignment will require students to apply the knowledge of FEM to analyze and solve a practical structural problem. The project must have sufficient engineering complexity conveying exciting knowledge or technical concepts to the entire class, giving students an appreciation of how powerful the method can be in performing structural analysis of real-world structures. The project can be either an individual work or students can work in groups of two or three. The project model can be selected by the students and approved by the lecturer, as it is in the students' best interest to propose projects that interest them.

The course should provide the students with the proper education to be able to use the most suitable finite element modeling methods and techniques to solve real structural analysis problems with a high degree of reliability. Further training on specific FEA software can be easily provided after graduation, through professional experience in design and consultancy firms.

It is evident that such a course cannot adequately cover all aspects of FEA education and cannot go into details about advanced topics such as plasticity, material and geometric nonlinearities, dynamic analysis, contact problems, coupled problems, modeling uncertainties and others. These topics are very broad and deep on their own and need to be covered in advanced tailored FEM postgraduate courses.

\section{Case Study: Sleipner A - North Sea oil Platform Collapse}

The construction of the original Sleipner A platform began in July 1989. The platform was to be used for oil and natural gas drilling in the Sleipender gas field in the North Sea (Tretiakova, 2012). It was built as a typical deep water structure platform with a concrete gravity base structure consisting of 24 cells, shown (partly completed) in Fig. 6, with a total base area of $16,000 \mathrm{~m}^{2}$. On 23 August 1991, the platform sank and collapsed during a regular controlled ballasting test in preparation for deck-mating in Gandsfjorden, outside Stavanger in Norway (Arnold, 2009). 


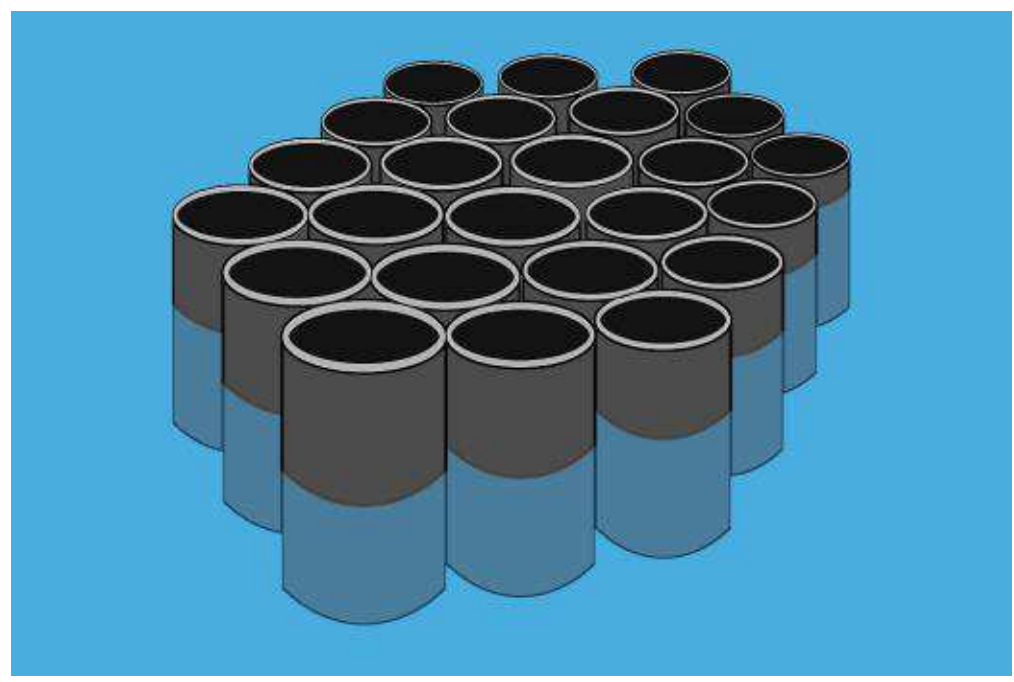

Fig. 6: 3D view of the buoyancy cells (partly completed) to be floated into the sea

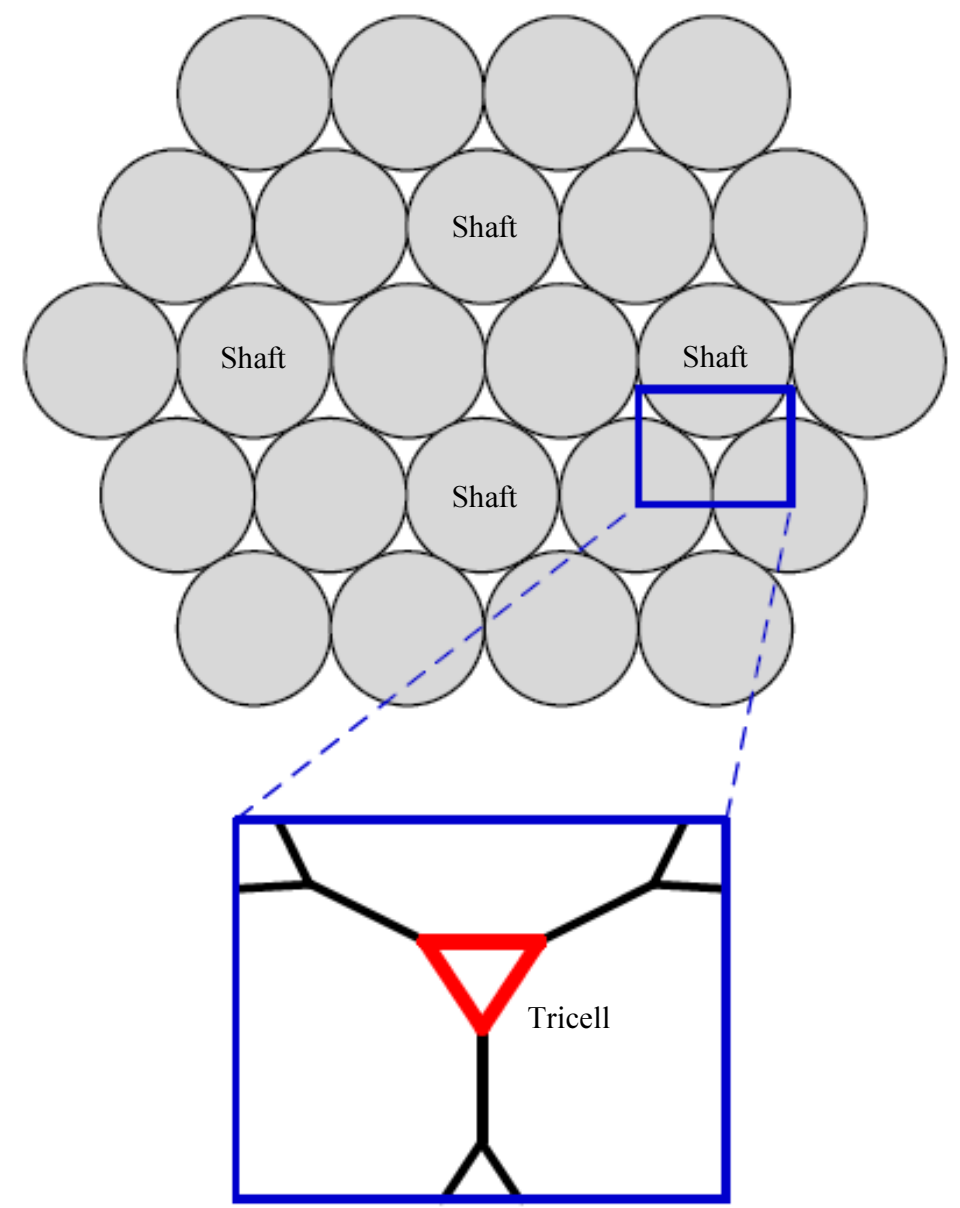

Fig. 7: Plan view of buoyancy cells with shafts and tricells highlighted

As the hull was lowered to the $65-\mathrm{m}$ mark, rumbling noises were heard followed by the sound of water pouring into the unit. A concrete cell had failed and a severe crack had formed, while seawater poured in at a very high rate. Within a few minutes, the hull began to sink very quickly. Finally, the structure sank 
deep into the $210 \mathrm{~m}$ sea, the buoyancy chambers imploded and the rubble struck the sea floor, creating an earthquake of magnitude 3 in the Richter scale. It took $18.5 \mathrm{~min}$ from the first sign of the accident until the platform disappeared from the surface of the sea (Jakobsen and Rosendahl, 1994).

Fortunately, no one was injured during the accident. A post-accident investigation was carried out by SINTEF in Norway which found out that the failure was due to a design flaw, that resulted from an error caused by unconservative concrete codes (Selby et al., 1997) and inaccurate FEA modeling of the tricell (Fig. 7), which formed part of the ballasting/flotation system (Arnold, 2009). The investigation traced the error to inaccurate finite element approximation of the linear elastic model of the tricell (using the FEA software NASTRAN) shown in Fig. 7.

It was concluded that the tricell walls and supports at the cell joints were the weakest points of the platform. Certain concrete walls were not thick enough to resist the hydrostatic pressure when the structure would be submerged. In addition, the finite element mesh used to analyze the tricells was proven to be too coarse to predict the shear stress distribution accurately. The shear stresses were underestimated by $47 \%$, leading to faulty design (Arnold, 2009). The most probable failure mechanism was found to be concrete crushing and shear failure that occurred in the portion of a tricell wall located above the transverse reinforcement, close to the joint.

Another possible flaw with the use of the software was the human error involved: The design software involved considerable complexity, which led to high perception of precision by the user. The software would only flag certain sections as critical and indicate them as sections that the engineers on the design team needed to check. However, the probable failure point of the platform was never flagged (Tretiakova, 2012) and as a consequence the design team never had the chance to investigate the issue more thoroughly.

The initial cost for the platform was around $\$ 180$ million. Statoil, the Norwegian multinational oil and gas company estimated their losses without the platform at about \$1 million per day. It took over two years to redesign the platform, build it and bring it to operational status, although the construction of the new platform was accomplished at an accelerated pace, reducing the schedule by 15 months, in comparison to the original structure (Jakobsen and Rosendahl, 1994).

\section{Conclusion}

FEA is a very demanding computational tool, as the analyst must be proficient not only in elasticity or mechanics, but also in mathematics, computer science and especially the finite element method itself. One aspect often overlooked when entering the finite element area is education. Training and experience in the computer software used are important and essential, but without adequate knowledge of the finite element method theory, a new user may fail miserably. This paper highlights this concern and the significance of error due to the lack of underlying knowledge by the FEA analyst while it attempts to make a useful contribution to the vital discussion on FEA engineering education at present.

Finite element computer programs have become standard tools in the hands of design engineers. The growing accessibility of FEA software brings with it the downside of ill-informed users relying on modeled results without having the advanced understanding needed to program the tests sufficiently well, or recognize the pitfalls where human or computer error could occur. While many software vendors have gone to great lengths to make their software accurate and easy to use, most users are not holding up their end by learning the techniques, engineering and discipline required to use these products successfully.

It cannot be emphasized strongly enough that while advanced FEA software programs produce vast amounts of results and colorful, highly persuasive pictures, it is the analyst's responsibility to ensure correctness and accuracy. FEA simulations are, in the end, approximate models and solutions, albeit highly sophisticated ones and it is the analyst's responsibility to ensure that results are valid and trustworthy. In the absence of such awareness, the system degenerates into a "black box" and the solution it provides will almost certainly be wrong, despite the impressive-looking results.

The case study presented highlights the potential of human error to undermine results. The Sleipner A failure shows the limitations of FEA and the risks involved in the careless use of it, highlighting the importance of sound and profound engineering education and knowledge about the theory behind FEA to obtain correct and reliable analysis results for designing realworld complex structures.

The key to good FE analysis is knowledge of the limitations of the method and an understanding of the physical phenomena under investigation. While computer-aided FEA makes a good engineer better, it can also make a bad engineer dangerous. In light of this, we believe that in the current context, a more rigorous approach to the teaching of FEA needs to be employed in university education.

\section{Author's Contributions}

Vagelis Plevris: Contributed in the conception and design of the research work, drafted the first versions of the article and made the figures used in the manuscript.

Gro Markeset: Contributed in the original conception and design of the research work, the design 
of the research plan, the organization of the study, the idea for the case study and the detailed review of the final version of the manuscript.

\section{Ethics}

This article is original and contains unpublished material. The corresponding author confirms that all the other authors have read and approved the manuscript and there are no ethical issues involved. The authors declare no conflict of interest.

\section{References}

Arnold, D.N., 2009. The sinking of the Sleipner a offshore platform.

Bandara, K.M.K., S.S. Bandara and M.T.R. Jayasinghe, 2009. Importance of accurate modeling input and assumptions in 3D finite element analysis of tall buildings. Engineer: J. Inst. Eng., 42: 5-10. DOI: 10.4038 /engineer.v42i2.7056

Gilewski, W., 2008. To teach or not to teach Finite Elements: This is the question. Proceedings of the 6th AECEF Symposium on Civil Engineering Education in Changing Europe, May 28-30, Vilnius, Lithuania.

Hibbeler, R.C., 1994. Mechanics of Materials. 2nd Edn.

Hossain, A., 2015. Teaching an undergraduate introductory finite element analysis course: Successful implementation for students learning.

Jakobsen, B. and F. Rosendahl, 1994. The sleipner platform accident. Struct. Eng. Int., 4: 190-103. DOI: $10.2749 / 101686694780601971$

Kurowski, P., 2002. When good engineers deliver bad FEA. http://machinedesign.com/fea-andsimulation/when-good-engineers-deliver-bad-fea
Macneal, R.H., 1993. Finite Elements: Their Design and Performance. 1st Edn., Marcel Dekker, Inc., New York, ISBN-10: 0824791622, pp: 531.

Nelson, T. and E. Wang, 2004. Reliable FE-modeling with ANSYS. Proceedings of the International ANSYS Conference, May 24-26, Pittsburgh, USA.

Plevris, V. and G. Tsiatas, 2018. Computational structural engineering: Past achievements and future challenges. Frontiers Built Environ., 4: 1-5. DOI: $10.3389 /$ fbuil.2018.00021

Selby, R.G., F.J. Vecchio and M.P. Collins, 1997. The failure of an offshore platform. Concrete Int., 19: 28-35.

Shaikh, F.U.A., 2012. Role of commercial software in teaching finite element analysis at undergraduate level: A case study. Eng. Educ., 7: 2-6. DOI: 10.11120/ened.2012.07020002

Timoshenko, S. and J.N. Goodier, 1951. Theory of Elasticity. 2nd Edn., McGraw-Hill, New York, pp: 506.

Tretiakova, K., 2012. Sleipner a - North Sea Oil Platform Collapse. https://failures.wikispaces.com/Sleipner+A++ North + Sea + Oil+Platform + Collapse

Young, B., E. Ellobody and T.W.C. Hu, 2012. 3D visualization of structures using finite-element analysis in teaching. J. Professional Issues Eng. Educ. Pract., 138: 131-138.

DOI: 10.1061/(ASCE)EI.1943-5541.0000093

Zamani, N.G., 2016. The challenges of teaching finite element analysis in the undergraduate curriculum. Proceedings of the 14th LACCEI International Multi-Conference for Engineering, Education and Technology: "Engineering Innovations for Global Sustainability", Jul. 20-22, San José, Costa Rica, pp: 1-10. 